\title{
Solvability in Distributions for a Class of Singular Differential Operators, III
}

By

\author{
Hidetoshi TAHARA*
}

We say that a linear partial differential equation $P u=f$ is locally solvable in $\mathscr{D}^{\prime}$ at $p$, if for any $f \in \mathscr{D}^{\prime}$ there exists a $u \in \mathscr{D}^{\prime}$ such that $P u=f$ holds near $p$. The following is one of the most fundamental problems: under what condition is $P u=f$ locally solvable in $\mathscr{D}^{\prime}$ ?

When $P$ is non-singular, this problem has been studied by many authors (for example, see the survey in $[6$, Chapter 26]). When $P$ is singular, in $[14,15,16]$ the author has established the local solvability in $\mathscr{D}^{\prime}$ for singular differential operators of various types: in [14] for operators of Fuchsian type, in [15] for operators of nonFuchsian hyperbolic type, and in [16] for operators of non-Fuchsian elliptic type.

In this paper, the author will establish the local solvability in $\mathscr{D}^{\prime}$ for a class of non-Fuchsian singular partial differential operators under much more general condition.

It should be noted that the following cases were already treated as to the local solvability for singular differential operators $P$. When $P$ is a Fuchsian operator of hyperbolic type, the solvability in $C^{\infty}, \mathscr{D}^{\prime}$ or $\mathscr{B}$ (where $\mathscr{B}$ means the set of all hyperfunctions) was discussed in $[1,2,3,4,5,11,12]$. When $P$ is a Fuchsian operator of elliptic type, the solvability in $\mathscr{B}$ was discussed in [9]. When $P$ is a non-Fuchsian operator of hyperbolic type, the solvability in $C^{\infty}$ was discussed in $[10,13]$. See also [7].

By the author's results (in $[14,15,16]$ and this paper), we can conclude that the class of operators for which the local solvability in $\mathscr{D}^{\prime}$ is valid is much wider than the class of Fuchsian operators.

\section{§1. Main Theorem}

Let $(t, x)=\left(t, x_{1}, \ldots, x_{n}\right) \in \mathbb{R}_{t} \times \mathbb{R}_{x}^{n}$ and let us consider

$$
P=\left(t \partial_{t}\right)^{m}+\sum_{\substack{j+|\alpha| \leqq m \\ j<m}} a_{j, \alpha}(t, x)\left(t \partial_{t}\right)^{j} \partial_{x}^{\alpha},
$$

where $m \in\{1,2, \ldots\}, \partial_{t}=\partial / \partial t, \partial_{x}=\left(\partial / \partial x_{1}, \ldots, \partial / \partial x_{n}\right), \alpha=\left(\alpha_{1}, \ldots, \alpha_{n}\right) \in\{0,1,2, \ldots\}^{n}$, $|\alpha|=\alpha_{1}+\cdots+\alpha_{n}, \partial_{x}^{\alpha}=\left(\partial / \partial x_{1}\right)^{\alpha_{1}} \ldots\left(\partial / \partial x_{n}\right)^{\alpha_{n}}$, and the coefficients $\mathrm{a}_{j, \alpha}(t, x)(j+|\alpha| \leqq m$ and $j<m)$ are $C^{\infty}$ functions defined in an open neighborhood $U$ of $(0,0)$ in $\mathbb{R}_{t} \times \mathbb{R}_{x}^{n}$.

Communicated by S. Matsuura, February 6, 1989.

* Department of Mathematics, Sophia University, Kioicho, Chiyoda-ku, Tokyo 102, Japan. 
Denote by $\mathscr{D}^{\prime}(U)$ the set of all distributions in $(t, x)$ defined on $U$. Put $p(\tau, \xi)$ and $\Sigma$ as follows:

$$
\begin{aligned}
& p(\tau, \xi)=\tau^{m}+\sum_{\substack{j+|\alpha|=m \\
j<m}} a_{j, \alpha}(0,0) \tau^{j} \xi^{\alpha}, \\
& \Sigma=\left\{(\tau, \xi) \in \mathbb{R}_{\tau} \times \mathbb{R}_{\xi}^{n} ; p(\tau, \xi)=0\right\} .
\end{aligned}
$$

Assume the following three conditions:

(A-1) When $j+|\alpha|=m, a_{j, \alpha}(t, x)$ is real-valued on $U$.

(A-2) $\quad \Sigma \cap\left\{(0, \xi) \in \mathbb{R}_{\tau} \times \mathbb{R}_{\xi}^{n} ; \xi \neq 0\right\}=\varnothing$.

(A-3) $\left(\frac{\partial p}{\partial \tau}\right)(\tau, \xi) \neq 0$, when $(0,0) \neq(\tau, \xi) \in \Sigma$.

Note that $P$ is not of Fuchsian type in $t$ (by (A-2) and (A-3)).

Then, we can state our main theorem as follows.

Theorem 1 . Let $P$ be the operator in (1.1). Assume (A-1), (A-2) and (A-3). Then, for any $f(t, x)(=f) \in \mathscr{D}^{\prime}(U)$ there exists a $u(t, x)(=u) \in \mathscr{D}^{\prime}(U)$ such that $P u=f$ holds near the origin $(0,0)$ in $\mathbb{R}_{t} \times \mathbb{R}_{x}^{n}$; that is, $P u=f$ is locally solvable in $\mathscr{D}^{\prime}$ at $(0,0)$.

As a special case, we have

Corollary. Let $P$ be the operator in (1.1). Assume (A-1) and the following: for any $\xi \in \mathbb{R}_{\xi}^{n} \backslash\{0\}$ the equation $p(\lambda, \xi)=0$ (in $\lambda \in \mathbb{C}$ ) has only simple and non-zero roots. Then, $P u=f$ is locally solvable in $\mathscr{D}^{\prime}$ at $(0,0)$.

Remark. More precisely, we can see the following result. For any $k \in \mathbb{Z}_{+}$ $(=\{0,1,2, \ldots\})$ there are $j_{k} \in \mathbb{Z}_{+}$and an open neighborhood $U_{k}$ of $(0,0)$ in $\mathbb{R}_{t} \times \mathbb{R}_{x}^{n}$ which satisfy the following: for any $f \in H^{-k}\left(U_{k}\right)$ there exists a $u \in H^{-j_{k}}\left(U_{k}\right)$ such that $P u=f$ holds on $U_{k}$. Here, $H^{-p}\left(U_{k}\right)$ denotes the usual Sobolev space on $U_{k}$.

Example. Our result can be applied to the following operators:

$$
P=\left(t \partial_{t}\right)^{2} \pm \Delta_{x}+a(t, x)\left(t \partial_{t}\right)+\left\langle b(t, x), \partial_{x}\right\rangle+c(t, x),
$$

where $\Delta_{x}$ is the Laplacian in $x$.

Let us compare the above result with the result for Fuchsian operators in [14], and let us make clear the difference between them. Let

$$
L=\left(t \partial_{t}\right)^{m}+\sum_{\substack{j+|\alpha| \leqq m \\ j<m}} a_{j, \alpha}(t, x)\left(t \partial_{t}\right)^{j}\left(t^{k} \partial_{x}\right)^{\alpha}
$$

where $\left(t^{k} \partial_{x}\right)^{\alpha}=\left(t^{k} \partial / \partial x_{1}\right)^{\alpha_{1}} \ldots\left(t^{k} \partial / \partial x_{n}\right)^{\alpha_{n}} \quad\left(=t^{k|\alpha|} \partial_{x}^{\alpha}\right)$. Denote by $\rho_{i}(x) \quad(1 \leqq i \leqq m)$ the roots of $\rho^{m}+\sum_{j<m} a_{j, 0}(0, x) \rho^{j}=0$. Define $p(\tau, \xi)$ as in (1.2) (where $a_{j, \alpha}(0,0)$ are the ones in (1.4)). Then, we already know the following result.

Theorem 2 (Fuchsian case: Tahara [14]). Let $L$ be the operator in (1.4). Assume $k \in\{1,2, \ldots\}, \rho_{i}(0) \notin\{-1,-2, \ldots\} \quad(1 \leqq i \leqq m),(\mathrm{A}-1)$ and the following: for any $\xi \in$ $\mathbb{R}_{\xi}^{n} \backslash\{0\}$ the equation $p(\lambda, \xi)=0$ (in $\lambda \in \mathbb{C}$ ) has only simple roots. Then, $L u=f$ is locally solvable in $\mathscr{D}^{\prime}$ at $(0,0)$. 
Note that $P$ in (1.1) corresponds to $L$ with $k=0$ and that the case $k=0$ is excluded from the consideration in Theorem 2 .

\section{§2. A Priori Estimates}

Before giving a proof of Theorem 1, let us establish here the following proposition.

Proposition 1. Let $P$ be the operator in (1.1), put

$$
P_{-s}=\left(t \partial_{t}-s\right)^{m}+\sum_{\substack{j+|\alpha| \leq m \\ j<m}} a_{j, \alpha}(t, x)\left(t \partial_{t}-s\right)^{j} \partial_{x}^{\alpha}
$$

for $s \in \mathbb{R}$, and let $\left(P_{-s}\right) *$ be the formal adjoint operator of $P_{-s}$. Assume (A-1) and (A-3). Then, there are $s_{k}>0\left(k \in \mathbb{Z}_{+}\right)$which satisfy the following: for any $k \in \mathbb{Z}_{+}$and any $s>s_{k}$ there are $\delta_{k, s}>0$ and an open neighborhood $V_{k, s}$ of $(0,0)$ in $\mathbb{R}_{t} \times \mathbf{R}_{x}^{n}$ such that the estimate

$$
\left\|\left(P_{-s}\right)^{*} \varphi\right\|_{k} \geqq \delta_{k, s}\left\|t^{m+k-1} \varphi\right\|_{m+k-1}
$$

holds for any $\varphi \in C_{0}^{\infty}\left(V_{k, s} \cap\{t>0\}\right)$ (or $\left.\varphi \in C_{0}^{\infty}\left(V_{k, s} \cap\{t<0\}\right)\right)$, where $\|w\|_{p}$ denotes the norm of $w$ in the Sobolev space $H^{p}\left(V_{k, s} \cap\{t>0\}\right)\left(\right.$ or $\left.H^{p}\left(V_{k, s} \cap\{t<0\}\right)\right)$.

First, we remark a fact on the decomposition of the following polynomial (in $\tau$ )

$$
\stackrel{\circ}{p}(t, x, \tau, \xi)=\tau^{m}+\sum_{\substack{j+|\alpha|=m \\ j<m}} a_{j, \alpha}(t, x) \tau^{j} \xi^{\alpha} .
$$

Let $W$ be a sufficiently small neighborhood of $(0,0)$ in $\mathbf{R}_{t} \times \mathbf{R}_{x}^{n}$. Then, by $(\mathrm{A}-1)$ and (A-3) we can see that all the real roots of the equation

$$
\stackrel{\circ}{p}(t, x, \lambda, \xi)=0 \quad(\text { in } \lambda \in \mathbb{C})
$$

are simple for any $(t, x, \xi) \in W \times\left(\mathbb{R}_{\xi}^{n} \backslash\{0\}\right)$, and that no roots of (2.3) change continuously from "real" to "non-real" when $(t, x, \xi)$ moves in $W \times\left(\mathbf{R}_{\xi}^{n} \backslash\{0\}\right)$. Therefore, denoting by $\lambda_{i}(t, x, \xi)(1 \leqq i \leqq p)$ the real roots of $(2.3)$ we have the following: (i) the number $p$ of the real roots of (2.3) is independent of $(t, x, \xi) \in W \times\left(\mathbb{R}_{\xi}^{n} \backslash\{0\}\right)$, (ii) $\lambda_{i}(t, x, \xi) \in C^{\infty}\left(W \times\left(\mathbb{R}_{\xi}^{n} \backslash\{0\}\right)\right)(1 \leqq i \leqq p)$, and (iii) $\lambda_{i}(t, x, \xi) \neq \lambda_{j}(t, x, \xi)$ for $1 \leqq i \neq j \leqq p$. Hence, by putting

$$
h(t, x, \tau, \xi)=\prod_{i=1}^{p}\left(\tau-\lambda_{i}(t, x, \xi)\right)
$$

we obtain a decomposition of $\stackrel{\circ}{p}(t, x, \tau, \xi)$ as follows:

$$
\stackrel{\circ}{p}(t, x, \tau, \xi)=h(t, x, \tau, \xi) e(t, x, \tau, \xi),
$$

where $e(t, x, \tau, \xi)$ has the form

$$
e(t, x, \tau, \xi)=\tau^{m-p}+\sum_{i=1}^{m-p} e_{i}(t, x, \xi) \tau^{m-p-i}
$$

and satisfies the following: (iv) $e_{i}(t, x, \xi) \in C^{\infty}\left(W \times\left(\mathbb{R}_{\xi}^{n} \backslash\{0\}\right)\right) \quad(1 \leqq i \leqq m-p)$, 
$e_{i}(t, x, \xi)$ is positively homogeneous of degree $i$ in $\xi$, and (vi) $e(t, x, \tau, \xi) \neq 0$ for any $(t, x, \tau, \xi) \in W \times \mathbb{R}_{\tau} \times\left(\mathbb{R}_{\xi}^{n} \backslash\{0\}\right)$.

Next, let us show two preparatory lemmas. In the discussion below, we use the following notation: $(t, x) \in[0, T] \times \mathbb{R}^{n}(T>0), D_{x}=-\sqrt{-1} \partial_{x},(w, v)$ denotes the inner product of $w$ and $v$ in $L^{2}\left((0, T) \times \mathbb{R}^{n}\right),\|w\|$ denotes the norm of $w$ in $L^{2}\left((0, T) \times \mathbb{R}^{n}\right)$, $\|w\|_{k}$ denotes the norm of $w$ in $H^{k}\left((0, T) \times \mathbb{R}^{n}\right)$, and

$$
\|\varphi\|_{k, s}=\sum_{i+|\alpha| \leqq k}\left\|\left(t \partial_{t}+s\right)^{i} \partial_{x}^{\alpha} \varphi\right\| .
$$

Obviously we can see the following: for any $k \in \mathbb{Z}_{+}$and $s \in \mathbb{R}$ there are $A_{k, s}>0$ and $B_{k, s}>0$ such that

$$
A_{k, s}\left\|t^{k} \varphi\right\|_{k} \leqq\|\varphi\|_{k, s} \leqq B_{k, s}\|\varphi\|_{k}
$$

holds for any $\varphi \in H^{k}\left((0, T) \times \mathbb{R}^{n}\right)$.

Lemma 1. Let

$$
H_{s}=\left(t \partial_{t}+s\right)^{p}+\sum_{i=1}^{p} a_{i}\left(t, x, D_{x}\right)\left(t \partial_{t}+s\right)^{p-i},
$$

where $a_{i}\left(t, x, D_{x}\right)(1 \leqq i \leqq p)$ are pseudo-differential operators with symbols $a_{i}(t, x, \xi)$ satisfying the following: (i) $a_{i}(t, x, \xi) \in C^{\infty}\left([0, T] \times \mathbb{R}_{x}^{n} \times \mathbb{R}_{\xi}^{n}\right)$, (ii) $a_{i}(t, x, \xi)$ is positively homogeneous of degree $i$ in $\xi($ for $|\xi| \geqq 1)$, and (iii) $a_{i}(t, x, \xi)$ is independent of $x$ for sufficiently large $|x|$. Assume that all the roots of the equation

$$
(\sqrt{-1} \lambda)^{p}+\sum_{i=1}^{p} a_{i}(t, x, \xi)(\sqrt{-1} \lambda)^{p-i}=0
$$

(in $\lambda$ ) are real and simple for any $(t, x, \xi) \in[0, T] \times \mathbb{R}_{x}^{n} \times \mathbb{R}_{\xi}^{n}$ satisfying $|\xi| \geqq 1$. Then, for any $k \in \mathbb{Z}_{+}$there are $b_{k}>0$ and $c_{k}>0$ such that the estimate

$$
\left\|H_{s} \varphi\right\|_{k, s} \geqq c_{k} s\|\varphi\|_{p+k-1, s}
$$

holds for any $\varphi \in C_{0}^{\infty}\left((0, T), H^{\infty}\left(\mathbb{R}^{n}\right)\right)$ and $s>b_{k}$.

Proof. We will prove this by reducing the problem to the one for a first-order system of pseudo-differential operators.

Denote by $\Lambda$ the pseudo-differential operator on $\mathbb{R}_{x}^{n}$ corresponding to the symbol $\left(1+|\xi|^{2}\right)^{1 / 2}$, by $\boldsymbol{S}^{k}([0, T])$ the set of all pseudo-differential operators of order $k$ on $\mathbb{R}_{x}^{n}$ depending smoothly on $t \in[0, T]$, and by $\mathcal{S}^{k}([0, T], p \times p)$ the set of all $p \times p$ matrices with components in $S^{k}([0, T])$. follows:

For $\varphi \in C_{0}^{\infty}\left((0, T), H^{\infty}\left(\mathbb{R}^{n}\right)\right)$ we put $v_{j} \in C_{0}^{\infty}\left((0, T), H^{\infty}\left(\mathbb{R}^{n}\right)\right)(j=0,1, \ldots, p-1)$ as

$$
v_{j}=(\sqrt{-1})^{p-j-1}\left(t \partial_{t}+s\right)^{j} \Lambda^{p-j-1} \varphi .
$$

Then, under the notations 


$$
\begin{aligned}
& h_{i}=(\sqrt{-1})^{i} a_{i}\left(t, x, D_{x}\right) \Lambda^{-i+1}, \quad i=1, \ldots, p,
\end{aligned}
$$

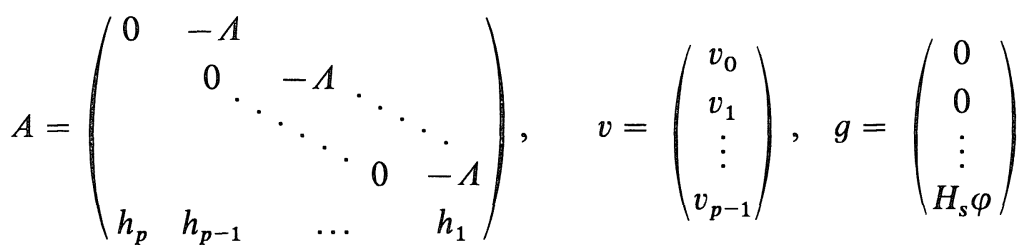

we have the following relation:

$$
\left(t \partial_{t}+s+\sqrt{-1} A\right) v=g .
$$

Moreover, by the standard argument for regularly hyperbolic systems (for example, see [8, Proposition 6.4]) we can see that there are $D \in \mathcal{S}^{1}([0, T], p \times p)$ and $M, N \in$ $S^{0}([0, T], p \times p)$ satisfying $D-D^{*} \in \mathcal{S}^{0}([0, T], p \times p), N A-D N \in \mathcal{S}^{0}([0, T], p \times p)$ and $M N-I \in \mathcal{S}^{-1}([0, T], p \times p)$.

Thus, to have Lemma 1 it is sufficient to show the following result: for any $k \in \mathbb{Z}_{+}$ there are $b_{k}>0$ and $c_{k}>0$ such that

$$
\sum_{i+j \leqq k}\left\|\left(t \partial_{t}+s\right)^{i} \Lambda^{j}\left(t \partial_{t}+s+\sqrt{-1} A\right) v\right\| \geqq c_{k} s \sum_{i+j \leqq k}\left\|\left(t \partial_{t}+s\right)^{i} \Lambda^{j} v\right\|
$$

holds for any $v \in C_{0}^{\infty}\left((0, T), H^{\infty}\left(\mathbf{R}^{n}\right)\right)^{p}$ and $s>b_{k}$.

Let us prove (2.7) from now. Take any $v \in C_{0}^{\infty}\left((0, T), H^{\infty}\left(\mathbf{R}^{n}\right)\right)^{p}$. Then we have

$$
N\left(t \partial_{t}+s+\sqrt{-1} A\right) v=\left(t \partial_{t}+s+\sqrt{-1} D\right) N v-t N_{t}^{\prime} v+\sqrt{-1}(N A-D N) v .
$$

Since the operators $t N_{t}^{\prime}, N A-D N$ and $D-D^{*}$ are bounded in $L^{2}\left((0, T) \times \mathbb{R}^{n}\right)^{p}$, we have

$$
\begin{aligned}
&\left\|N\left(t \partial_{t}+s+\sqrt{-1} A\right) v\right\|^{2} \\
& \geqq \frac{1}{2}\left\|\left(t \partial_{t}+s+\sqrt{-1} D\right) N v\right\|^{2}-C_{1}\|v\|^{2} \\
&= \frac{1}{2}\left\|\left(t \partial_{t}+\sqrt{-1} D\right) N v\right\|^{2}+\frac{s^{2}}{2}\|N v\|^{2} \\
& \quad+s \operatorname{Re}\left(\left(t \partial_{t}+\sqrt{-1} D\right) N v, N v\right)-C_{1}\|v\|^{2} \\
& \geqq \frac{1}{2}\left\|\left(t \partial_{t}+\sqrt{-1} D\right) N v\right\|^{2}+\frac{s^{2}}{2}\|N v\|^{2}-s C_{2}\|N v\|^{2}-C_{1}\|v\|^{2}
\end{aligned}
$$

for some $C_{1}>0$ and $C_{2}>0$. Therefore, if $s>4 C_{2}$, we obtain

$$
\left\|N\left(t \partial_{t}+s+\sqrt{-1} A\right) v\right\|^{2} \geqq \frac{s^{2}}{4}\|N v\|^{2}-C_{1}\|v\|^{2} .
$$

On the other hand, since

$$
\Lambda^{-1}\left(t \partial_{t}+s+\sqrt{-1} A\right) v=\left(t \partial_{t}+s\right) \Lambda^{-1} v+\sqrt{-1} \Lambda^{-1} \cdot A v
$$

holds and since $\Lambda^{-1} A$ is bounded in $L^{2}\left((0, T) \times \mathbb{R}^{n}\right)^{p}$, for $s>1 / 2$ we have 


$$
\begin{aligned}
& \left\|\Lambda^{-1}\left(t \partial_{t}+s+\sqrt{-1} A\right) v\right\|^{2} \\
& \geqq \frac{1}{2}\left\|\left(t \partial_{t}+s\right) \Lambda^{-1} v\right\|^{2}-C_{3}\|v\|^{2} \\
& =\frac{1}{2}\left\|t \partial_{t} \Lambda^{-1} v\right\|^{2}+\frac{s^{2}-s}{2}\left\|\Lambda^{-1} v\right\|^{2}-C_{3}\|v\|^{2} \\
& \geqq \frac{s^{2}}{4}\left\|\Lambda^{-1} v\right\|^{2}-C_{3}\|v\|^{2}
\end{aligned}
$$

for some $C_{3}>0$. Hence, by (2.8) and (2.9) we obtain

$$
\begin{aligned}
& \left\|N\left(t \partial_{t}+s+\sqrt{-1} A\right) v\right\|^{2}+\left\|\Lambda^{-1}\left(t \partial_{t}+s+\sqrt{-1} A\right) v\right\|^{2} \\
& \geqq \frac{s^{2}}{4}\left(\|N v\|^{2}+\left\|\Lambda^{-1} v\right\|^{2}\right)-\left(C_{1}+C_{3}\right)\|v\|^{2} .
\end{aligned}
$$

Here, we note the following: there are $C_{4}>0$ and $C_{5}>0$ such that

$$
C_{4}\|w\|^{2} \leqq\|N w\|^{2}+\left\|\Lambda^{-1} w\right\|^{2} \leqq C_{5}\|w\|^{2}
$$

holds for any $w \in C_{0}^{\infty}\left((0, T), H^{\infty}\left(\mathbb{R}^{n}\right)\right)^{p}$. In fact, this is verified by $\|N w\|+\left\|\Lambda^{-1} w\right\| \leqq$ $\left(\|N\|+\left\|\Lambda^{-1}\right\|\right)\|w\|$ and $\|w\| \leqq\|M N w\|+\|(I-M N) w\| \leqq\|M\|\|N w\|+\|(I-M N) \Lambda\| \cdot$ $\left\|\Lambda^{-1} w\right\| \leqq(\|M\|+\|(I-M N) \Lambda\|)\left(\|N w\|+\left\|\Lambda^{-1} w\right\|\right)$.

Therefore, by (2.10) and (2.11) we have

$$
C_{5}\left\|\left(t \partial_{t}+s+\sqrt{-1} A\right) v\right\|^{2} \geqq\left(\frac{s^{2}}{4} C_{4}-C_{1}-C_{3}\right)\|v\|^{2} .
$$

Thus, by choosing $b_{0}=\max \left\{4 C_{2}, 1 / 2,8\left(C_{1}+C_{3}\right) / C_{4}\right\}$ and $c_{0}=\left(C_{4} / 8 C_{5}\right)^{1 / 2}$ we obtain

$$
\left\|\left(t \partial_{t}+s+\sqrt{-1} A\right) v\right\| \geqq c_{0} s\|v\|
$$

for $s>b_{0}$. Thus, we have proved (2.7) for $k=0$.

Note that

$$
\left\{\begin{array}{l}
\Lambda\left(t \partial_{t}+s+\sqrt{-1} A\right) v=\left(t \partial_{t}+s+\sqrt{-1} A+\sqrt{-1}[\Lambda, A] \Lambda^{-1}\right) \Lambda v \\
\left(t \partial_{t}+s\right)\left(t \partial_{t}+s+\sqrt{-1} A\right) v=\left(t \partial_{t}+s+\sqrt{-1} A\right)\left(t \partial_{t}+s\right) v+\sqrt{-1}\left(t A_{t}^{\prime} \Lambda^{-1}\right) \Lambda v
\end{array}\right.
$$

hold and that $[\Lambda, A] \Lambda^{-1}, t A_{t}^{\prime} \Lambda^{-1} \in \mathcal{S}^{0}([0, T], p \times p)$ are bounded in $L^{2}\left((0, T) \times \mathbb{R}^{n}\right)^{p}$. Therefore, by using (2.12) and by induction on $k$ we can prove (2.7) for $k \geqq 1$ in the same way as above.

Q.E.D.

Lemma 2. Let

$$
E_{s}=\left(t \partial_{t}+s\right)^{q}+\sum_{i=1}^{q} a_{i}\left(t, x, D_{x}\right)\left(t \partial_{t}+s\right)^{q-i}
$$

where $a_{i}\left(t, x, D_{x}\right)(1 \leqq i \leqq q)$ are pseudo-differential operators with symbols $a_{i}(t, x, \xi)$ satisfying the following: (i) $a_{i}(t, x, \xi) \in C^{\infty}\left([0, T] \times \mathbb{R}_{x}^{n} \times \mathbb{R}_{\xi}^{n}\right)$, (ii) $a_{i}(t, x, \xi)$ is positively homogeneous of degree $i$ in $\xi($ for $|\xi| \geqq 1)$, and (iii) $a_{i}(t, x, \xi)$ is independent of $x$ for sufficiently large $|x|$. Assume that 


$$
(\sqrt{-1} \tau)^{q}+\sum_{i=1}^{q} a_{i}(0,0, \xi)(\sqrt{-1} \tau)^{q-i} \neq 0
$$

holds for any $(\tau, \xi) \in \mathbb{R}_{\tau} \times \mathbb{R}_{\xi}^{n}$ satisfying $|\xi| \geqq 1$. Then, there are an open neighborhood $W$ of $(0,0)$ in $\mathbf{R}_{t} \times \mathbb{R}_{x}^{n}, d_{k}>0\left(k \in \mathbb{Z}_{+}\right)$and $C_{k, s}>0\left(k \in \mathbb{Z}_{+}\right.$and $\left.s \in \mathbf{R}\right)$ such that the estimate

$$
\left\|E_{s} \varphi\right\|_{k, s} \geqq d_{k}\|\varphi\|_{q+k, s}-C_{k, s}\|\varphi\|_{q+k-1, s}
$$

holds for any $\varphi \in C_{0}^{\infty}(W \cap\{t>0\}), k \in \mathbb{Z}_{+}$and $s \in \mathbb{R}$.

Proof. Put $B(r)=\left\{x \in \mathbb{R}^{n} ;|x|<r\right\}$, and assume that $\varphi(t, x)(=\varphi) \in C_{0}^{\infty}((0, \varepsilon) \times B(r))$. Let $\mu_{r}(x) \in C_{0}^{\infty}(B(2 r))$ be such that $\mu_{r}=1$ holds on $B(r)$. Obviously we have $\varphi=\mu_{r} \varphi$.

Put

$$
E_{s}^{(0,0)}=\left(t \partial_{t}+s\right)^{q}+\sum_{i=1}^{q} a_{i}\left(0,0, D_{x}\right)\left(t \partial_{t}+s\right)^{q-i}
$$

and choose $b_{i}\left(t, x, D_{x}\right), c_{i j}\left(t, x, D_{x}\right) \in S^{i}([0, T])$ so that the following relation holds:

$$
a_{i}\left(t, x, D_{x}\right)=a_{i}\left(0,0, D_{x}\right)+t b_{i}\left(t, x, D_{x}\right)+\sum_{j=1}^{n} x_{j} c_{i j}\left(t, x, D_{x}\right) .
$$

Then, for $\varphi \in C_{0}^{\infty}((0, \varepsilon) \times B(r))$ we have

$$
\begin{aligned}
E_{s} \varphi= & E_{s}^{(0,0)} \varphi+\sum_{i=1}^{q} t b_{i}\left(t \partial_{t}+s\right)^{q-i} \varphi \\
& +\sum_{i=1}^{q} \sum_{j=1}^{n}\left(x_{j} \mu_{r}\right) c_{i j}\left(t \partial_{t}+s\right)^{q-i} \varphi \\
& +\sum_{i=1}^{q} \sum_{j=1}^{n} x_{j}\left[c_{i j}, \mu_{r}\right]\left(t \partial_{t}+s\right)^{q-i} \varphi .
\end{aligned}
$$

Therefore, by the conditions $|t| \leqq \varepsilon, \quad\left|x_{j} \mu_{r}(x)\right| \leqq 2 r$ and $\left[c_{i j}, \mu_{r}\right] \quad\left(=c_{i j} \mu_{r}-\mu_{r} c_{i j}\right) \in$ $S^{i-1}([0, T])$ we obtain

$$
\left\|E_{s} \varphi\right\| \geqq\left\|E_{s}^{(0,0)} \varphi\right\|-(\varepsilon+r) A_{1}\|\varphi\|_{q, s}-C_{r}\|\varphi\|_{q-1, s}
$$

for some $A_{1}>0$ (independent of $\varepsilon$ and $r$ ) and $C_{r}>0$ (depending on $r$ ). Thus, in order to estimate $\left\|E_{s} \varphi\right\|$ from below we need to estimate $\left\|E_{s}^{(0,0)} \varphi\right\|$ from below.

Note the following fact. Put

$$
R=\partial_{z}^{q}+\sum_{i=1}^{q} a_{i}\left(0,0, D_{x}\right) \partial_{z}^{q-i}
$$

Then, by (2.13) and by using the Fourier transformation we can see the following: there are $\mu>0$ and $A_{2}>0$ such that

$$
\|R \psi\|_{(z, x)} \geqq \mu \sum_{i+|\alpha| \leqq q}\left\|\partial_{z}^{i} \partial_{x}^{\alpha} \psi\right\|_{(z, x)}-A_{2} \sum_{i+|\alpha| \leqq q-1}\left\|\partial_{z}^{i} \partial_{x}^{\alpha} \psi\right\|_{(z, x)}
$$

holds for any $\psi=\psi(z, x) \in C_{0}^{\infty}\left(\mathbf{R}_{z} \times \mathbb{R}_{x}^{n}\right)$, where $\|w\|_{(z, x)}$ is the norm of $w$ in $L^{2}\left(\mathbb{R}_{z} \times \mathbf{R}_{x}^{n}\right)$.

By using (2.16), let us estimate $\left\|E_{s}^{(0,0)} \varphi\right\|$ from below. Note the following: by the change of variables $(0, T) \times \mathbf{R}_{x}^{n} \ni(t, x) \rightarrow(z, x)=(\log t, x) \in \mathbf{R}_{z} \times \mathbf{R}_{x}^{n}, t \partial_{t}$ is transformed 
into $\partial_{z}, E_{0}^{(0,0)}$ is transformed into $R, \phi(t, x) \in \mathbb{C}_{0}^{\infty}\left((0, T) \times \mathbb{R}_{x}^{n}\right)$ is transformed into $\psi(z, x)=\phi\left(e^{z}, x\right) \in C_{0}^{\infty}\left(\mathbb{R}_{z} \times \mathbb{R}_{x}^{n}\right)$, and $d t / t$ is transformed into $d z$. Therefore, by (2.16) we have

$$
\left\|\frac{1}{\sqrt{t}} E_{0}^{(0,0)} \phi\right\| \geqq \mu \sum_{i+|\alpha| \leq q}\left\|\frac{1}{\sqrt{t}}\left(t \partial_{t}\right)^{i} \partial_{x}^{\alpha} \phi\right\|-A_{2} \sum_{i+|\alpha| \leq q-1}\left\|\frac{1}{\sqrt{t}}\left(t \partial_{t}\right)^{i} \partial_{x}^{\alpha} \phi\right\|
$$

for any $\phi=\phi(t, x) \in C_{0}^{\infty}\left((0, T) \times \mathbb{R}_{x}^{n}\right)$. Moreover, by putting $\phi(t, x)=\sqrt{t} \varphi(t, x)$ we obtain

$$
\left\|E_{1 / 2}^{(0,0)} \varphi\right\| \geqq \mu\|\varphi\|_{q, 1 / 2}-A_{2}\|\varphi\|_{q-1,1 / 2}
$$

for any $\varphi \in C_{0}^{\infty}\left((0, T) \times \mathbb{R}_{x}^{n}\right)$. Since

$$
\left|\|\varphi\|_{l, s}-\|\varphi\|_{l, 1 / 2}\right| \leqq C_{l, s}\|\varphi\|_{l-1, s}
$$

holds for some $C_{l, s}>0$, by (2.17) and (2.18) we obtain

$$
\begin{aligned}
\left\|E_{s}^{(0,0)} \varphi\right\| & \geqq\left\|E_{1 / 2}^{(0,0)} \varphi\right\|-\left\|\left(E_{s}^{(0,0)}-E_{1 / 2}^{(0,0)}\right) \varphi\right\| \\
& \geqq \mu\|\varphi\|_{q, 1 / 2}-A_{2}\|\varphi\|_{q-1,1 / 2}-A_{3, s}\|\varphi\|_{q-1, s} \\
& \geqq \mu\|\varphi\|_{q, s}-B_{s}\|\varphi\|_{q-1, s}
\end{aligned}
$$

for some $A_{3, s}>0$ and $B_{s}>0$ (depending on $s$ ).

Hence, by (2.15) and (2.19) we have

$$
\left\|E_{s} \varphi\right\| \geqq\left(\mu-(\varepsilon+r) A_{1}\right)\|\varphi\|_{q, s}-\left(B_{s}+C_{r}\right)\|\varphi\|_{q-1, s}
$$

for any $\varphi \in C_{0}^{\infty}((0, \varepsilon) \times B(r))$. Thus, by putting $\varepsilon=1 / 4 A_{1}, r=1 / 4 A_{1}, W=(-\varepsilon, \varepsilon) \times$ $B(r), d_{0}=\mu / 2$ and $C_{0, s}=B_{s}+C_{r}$ we can obtain

$$
\left\|E_{s} \varphi\right\| \geqq d_{0}\|\varphi\|_{q, s}-C_{0, s}\|\varphi\|_{q-1, s} .
$$

Thus, we have proved (2.14) for $k=0$. (2.14) for $k \geqq 1$ may be proved by induction on $k$.

Q.E.D.

Now, by using Lemmas 1 and 2 let us give a proof of Proposition 1 .

Proof of Proposition 1. Let $P_{-s}$ be as in (2.1). Then, for any $\varphi \in C^{\infty}(U)$ we have

$$
\begin{aligned}
\left(P_{-s}\right)^{*} \varphi & =\left(-t \partial_{t}-1-s\right)^{m}+\sum_{j+|\alpha| \leq m}\left(-t \partial_{t}-1-s\right)^{j}\left(-\partial_{x}\right)^{\alpha} a_{j, \alpha}(t, x) \varphi \\
& =(-1)^{m}\left[\left(t \partial_{t}+s\right)^{m}+\sum_{\substack{j+|\alpha| \leq m \\
j<m}} b_{j, \alpha}(t, x)\left(t \partial_{t}+s\right)^{j} \partial_{x}^{\alpha}\right] \varphi
\end{aligned}
$$

for some $b_{j, \alpha}(t, x) \in C^{\infty}(U)$ such that $b_{j, \alpha}(t, x)=a_{j, \alpha}(t, x)$ for $j+|\alpha|=m$ and therefore

$$
\tau^{m}+\sum_{\substack{j+|\alpha|=m \\ j<m}} b_{j, \alpha}(0,0) \tau^{j} \xi^{\alpha}=p(\tau, \xi) .
$$

Since we are discussing $\left(P_{-s}\right)^{*}$ only in a small neighborhood of $(0,0)$ in $\mathbb{R}_{t} \times \mathbb{R}_{x}^{n}$, we may assume that $b_{j, \alpha}(t, x)$ is constant outside a small neighborhood of $(0,0)$ in $\mathbb{R}_{t} \times \mathbb{R}_{x}^{n}$. 
Then, by $(2.4),(\mathrm{A}-1)$ and $(\mathrm{A}-3)$ we can see that $\left(P_{-s}\right) *$ is decomposed into the following form:

$$
\left(P_{-s}\right)^{*}=(-1)^{m} H_{s} E_{s}+\sum_{i=1}^{m-1} Q_{i}\left(t, x, D_{x}\right)\left(t \partial_{t}+s\right)^{m-1-i}
$$

where $H_{s}$ is an operator of order $p$ satisfying the conditions in Lemma $1, E_{s}$ is an operator of order $q(=m-p)$ satisfying the conditions in Lemma 2, and $Q_{i}\left(t, x, D_{x}\right) \in$ $S^{i}([0, T])(1 \leqq i \leqq m-1)$.

Choose $b_{k}, c_{k}, W, d_{k}$ and $C_{k, s}$ so that the conditions in Lemmas 1 and 2 hold for the operators $H_{s}$ and $E_{s}$ in (2.20). Let $k \in \mathbb{Z}_{+}$. Then, by choosing a constant $M_{k}>0$ suitably we have

$$
\begin{aligned}
& \left\|\left(P_{-s}\right)^{*} \varphi\right\|_{k, s} \\
& \quad \geqq\left\|H_{s}\left(E_{s} \varphi\right)\right\|_{k, s}-M_{k}\|\varphi\|_{m+k-1, s} \\
& \quad \geqq c_{k} s\left\|E_{s} \varphi\right\|_{p+k-1, s}-M_{k}\|\varphi\|_{m+k-1, s} \\
& \quad \geqq c_{k} s\left(d_{p+k-1}\|\varphi\|_{m+k-1, s}-C_{p+k-1, s}\|\varphi\|_{m+k-2, s}\right)-M_{k}\|\varphi\|_{m+k-1, s}
\end{aligned}
$$

for any $\varphi \in C_{0}^{\infty}(W \cap\{t>0\})$ and $s>b_{k}$.

Here, we put $W(r)=\left\{(t, x) \in W ;\left|x_{i}\right|<(r / \sqrt{2})(i=1, \ldots, n)\right\}$ and note the following: if $\varphi \in C_{0}^{\infty}(W(r) \cap\{t>0\})$, we have

$$
\|\varphi\|_{m+k-2, s} \leqq r\|\varphi\|_{m+k-1, s}
$$

by using Poincare's inequality with respect to the $x$-variable.

Therefore, by (2.21) and (2.22) we have

$$
\begin{aligned}
\left\|\left(P_{-s}\right)^{*} \varphi\right\|_{k, s} \geqq & \left(\frac{c_{k} d_{p+k-1}}{2} s-M_{k}\right)\|\varphi\|_{m+k-1, s} \\
& +c_{k} s\left(\frac{d_{p+k-1}}{2}-r C_{p+k-1, s}\right)\|\varphi\|_{m+k-1, s} .
\end{aligned}
$$

Hence, by putting $s_{k}=\left(4 M_{k}\right) /\left(c_{k} d_{p+k-1}\right), r_{k, s}=d_{p+k-1} /\left(4 C_{p+k-1, s}\right), V_{k, s}=W\left(r_{k, s}\right)$ and by taking $s>s_{k}$ we have

$$
\left\|\left(P_{-s}\right)^{*} \varphi\right\|_{k, s} \geqq \frac{c_{k} d_{p+k-1}}{2} s\|\varphi\|_{m+k-1, s}
$$

for any $\varphi \in C_{0}^{\infty}\left(V_{k, s} \cap\{t>0\}\right)$. Thus, by (2.6) and (2.23) we can obtain (2.2). Q.E.D.

\section{§3. Proof of Theorem 1}

As in [14], we put $\mathscr{D}_{0}^{\prime}, \mathscr{D}^{\prime}(+), \mathscr{D}^{\prime}(-), \mathscr{D}_{\{t=0\}}^{\prime}, \mathscr{D}_{\text {ext }}^{\prime}(+)$ and $\mathscr{D}_{\text {ext }}^{\prime}(-)$ as follows:

$$
\begin{aligned}
& \mathscr{D}_{0}^{\prime}=\underset{W_{\ni}(0,0)}{\text { ind }-\lim _{1}} \mathscr{D}^{\prime}(W), \\
& \mathscr{D}^{\prime}( \pm)=\underset{W \ni(0,0)}{\underset{\text { ind }-\lim _{\longrightarrow}}{\longrightarrow}} \mathscr{D}^{\prime}(W \cap\{ \pm t>0\}),
\end{aligned}
$$




$$
\begin{aligned}
\mathscr{D}_{\{t=0\}}^{\prime} & =\left\{u \in \mathscr{D}_{0}^{\prime} ; \operatorname{supp}(u) \subset\{t=0\}\right\}, \\
\mathscr{D}_{\text {ext }}^{\prime}( \pm) & =\left\{u \in \mathscr{D}^{\prime}( \pm) ; \text { there exists a } v \in \mathscr{D}_{0}^{\prime} \text { such that } u=v \text { on }\{ \pm t>0\}\right\}
\end{aligned}
$$

where $W$ is an open neighborhood of $(0,0)$ in $\mathbb{R}_{t} \times \mathbb{R}_{x}^{n}$. Note that $\mathscr{D}_{\text {ext }}^{\prime}( \pm)$ is the set of all distributions $u \in \mathscr{D}^{\prime}( \pm)$ which is extendable to a full neighborhood of $t=0$ as a distribution.

Then, we can see that Theorem 1 is obtained by the following two facts:

(S-1) $\quad P u=f$ is solvable in $\mathscr{D}_{\{t=0\}}^{\prime}$.

(S-2) $\quad P u=f$ is solvable in $\mathscr{D}_{\text {ext }}^{\prime}( \pm)$.

In fact, if we know (S-1) and (S-2), the solvability of $P u=f$ in $\mathscr{D}_{0}^{\prime}$ is obtained by the following commutative diagram:

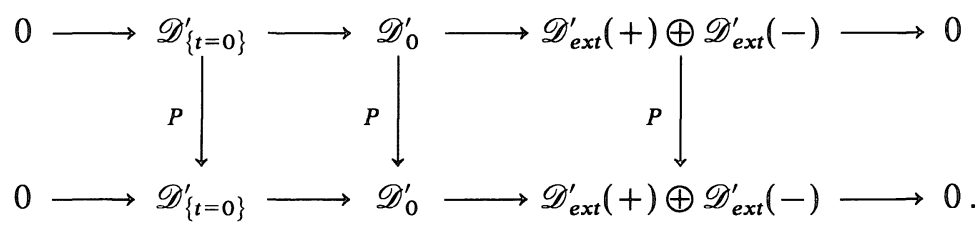

Note that the horizontal line is exact, since for any $u \in \mathscr{D}_{\text {ext }}^{\prime}( \pm)$ we can find a $v \in \mathscr{D}_{0}^{\prime}$ such that $u=v$ on $\{ \pm t>0\}$ and $\operatorname{supp}(v) \subset\{ \pm t \geqq 0\}$.

Hence to have Theorem 1 it is sufficient to prove (S-1) and (S-2).

Proof of $(S-1)$. Put

$$
C\left(\rho, x, \partial_{x}\right)=\rho^{m}+\sum_{\substack{j+|\alpha| \leqq m \\ j<m}} a_{j, \alpha}(0, x) \rho^{j} \partial_{x}^{\alpha} .
$$

Let $u$ and $f$ be of the form

$$
u=\sum_{i=0}^{N} \delta^{(i)}(t) \otimes \psi_{i}(x), \quad f=\sum_{i=0}^{N} \delta^{(i)}(t) \otimes \mu_{i}(x),
$$

where $N \in \mathbb{Z}_{+}, \delta^{(i)}(t)=\partial_{t}^{i} \delta(t), \delta(t)=\delta^{(0)}(t)$ is Dirac's delta-function, and $\psi_{i}(x), \mu_{i}(x)$ are germs of distributions in $x$ at the origin in $\mathbb{R}_{x}^{n}$. Then, by using the relations

$$
\left(t \partial_{t}\right)^{k} \delta^{(i)}(t)=(-i-1)^{k} \delta^{(i)}(t) \quad\left(k, i \in \mathbb{Z}_{+}\right)
$$

we can see that $P u=f$ is equivalent to the following recursive system:

$$
\left\{\begin{array}{l}
C\left(-N-1 ; x, \partial_{x}\right) \psi_{N}=\mu_{N}, \\
C\left(-N ; x, \partial_{x}\right) \psi_{N-1}=\mu_{N-1}+L_{N-1, N}\left(x, \partial_{x}\right) \psi_{N}, \\
\vdots \vdots \vdots \vdots \vdots \vdots \vdots \vdots \\
C\left(-1, x, \partial_{x}\right) \psi_{0}=\mu_{0}+\sum_{l=1}^{N} L_{0, l}\left(x, \partial_{x}\right) \psi_{l},
\end{array}\right.
$$

where $L_{i, l}\left(x, \partial_{x}\right)(0 \leqq i \leqq N-1$ and $i+1 \leqq l \leqq N)$ are differential operators of order $m$ determined by $P$. Since $C\left(\rho, x, \partial_{x}\right)$ is assumed to be elliptic near $x=0$ (by (A-2)), we 
know that the equation $C\left(\rho, x, \partial_{x}\right) \psi=\mu$ (where $\psi=\psi(x), \mu=\mu(x)$ are distributions in $x$ near $x=0$ ) is solvable in the germ sense. Therefore, by solving (3.2) successively we can determine $\left\{\psi_{i}\right\}_{i=0}^{N}$ from the given $\left\{\mu_{i}\right\}_{i=0}^{N}$ so that $P u=f$ holds under (3.1). This proves (S-1), because any $u, f \in \mathscr{D}_{\{t=0\}}^{\prime}$ are expressed in the form (3.1). Q.E.D.

Proof of (S-2). Let $f \in \mathscr{D}_{\text {ext }}^{\prime}(+)$. Then we have $f \in H^{-m-k+1}(V \cap\{t>0\})$ for some $k \in \mathbb{Z}_{+}$and some open neighborhood $V$ of $(0,0)$ in $\mathbf{R}_{t} \times \mathbb{R}_{x}^{n}$. Let $s_{k}$ be the one in Proposition 1. Choose $s \in \mathbb{Z}$ satisfying $s>\max \left\{s_{k}, m+k-1\right\}$ and fix it. Let $\delta_{k, s}$ and $V_{k, s}$ be the ones in Proposition 1 corresponding to these $k$ and s. Put $W=V \cap V_{k, s}$. Then, we can see the following two facts:

$$
\begin{gathered}
t^{s-m-k+1} f \in H^{-m-k+1}(W \cap\{t>0\}), \\
\left\|\left(P_{-s}\right)^{*} \varphi\right\|_{k} \geqq \delta_{k, s}\left\|t^{m+k-1} \varphi\right\|_{m+k-1} \quad \text { for any } \varphi \in C_{0}^{\infty}(W \cap\{t>0\}) .
\end{gathered}
$$

Let $H_{0}^{k}(W \cap\{t>0\})$ be the closure of $C_{0}^{\infty}(W \cap\{t>0\})$ in the Sobolev space $H^{k}(W \cap$ $\{t>0\})$, define a linear subspace $Z$ of $H_{0}^{k}(W \cap\{t>0\})$ by $Z=\left\{\left(P_{-s}\right)^{*} \varphi ; \varphi \in C_{0}^{\infty}(W \cap\right.$ $\{t>0\})\}$, and define a linear functional $T$ on $Z$ by $T\left(\left(P_{-s}\right)^{*} \varphi\right)=\left\langle\varphi, t^{s} f\right\rangle$. Then, by (3.3) and (3.4) we have

$$
\begin{aligned}
\left|T\left(\left(P_{-s}\right)^{*} \varphi\right)\right| & =\left|\left\langle t^{m+k-1} \varphi, t^{s-m-k+1} f\right\rangle\right| \\
& \leqq\left\|t^{m+k-1} \varphi\right\|_{m+k-1}\left\|t^{s-m-k+1} f\right\|_{-m-k+1} \\
& \leqq \frac{1}{\delta_{k, s}}\left\|\left(P_{-s}\right)^{*} \varphi\right\|_{k}\left\|t^{s-m-k+1} f\right\|_{-m-k+1},
\end{aligned}
$$

and therefore $T$ is continuous on $Z$ with respect to the topology induced from $H_{0}^{k}(W \cap$ $\{t>0\})$. Since $H^{-k}(W \cap\{t>0\})$ is the dual space of $H_{0}^{k}(W \cap\{t>0\})$, we can find a $v \in H^{-k}(W \cap\{t>0\})$ such that $T(z)=\langle z, v\rangle$ for any $z \in Z$. This means that $\left\langle\varphi, t^{s} f\right\rangle=$ $\left\langle\left(P_{-s}\right)^{*} \varphi, v\right\rangle$ holds for any $\varphi \in C_{0}^{\infty}(W \cap\{t>0\})$. Hence, we have $P_{-s} v=t^{s} f$ on $W \cap$ $\{t>0\}$; this is equivalent to $P\left(t^{-s} v\right)=f$ on $W \cap\{t>0\}$. Thus, by putting $u=t^{-s} v$ we obtain a solution $u \in \mathscr{D}_{\text {ext }}^{\prime}(+)$ of $P u=f$.

Q.E.D.

\section{References}

[1] Alinhac, S., Systèmes hyperboliques singuliers. Asterisque, 19 (1974), 3-24.

[2] Bove, A., Lewis, J. E. and Parenti, C., Cauchy problem for Fuchsian hyperbolic operators, Hokkaido Math. J., 14 (1985), 175-248.

[3] $\longrightarrow$, Structure properties of solutions of some Fuchsian hyperbolic equations, Math. Ann., 273 (1986), 553-571.

[4] Bove, A., Lewis, J. E., Parenti, C. and Tahara, H., Cauchy problem for Fuchsian hyperbolic operators, II. J. Fac. Sci. Univ. Tokyo Sect. IA Math., 34 (1987), 127-157.

[ 5 ] Hanges, N., Parametrices and local solvability for a class of singular hyperbolic operators, Comm. in PDE, 3 (1978), 105-152.

[6] Hörmander, L., The analysis of linear partial differential operators, IV. Springer, 1985.

[7] Ivrii, V. Ja., Wave fronts of solutions of certain pseudodifferential equations, Trudy Moscow Mat., 39 (1979), 49-85.

[ 8 ] Mizohata, S., The theory of partial differential equations, Cambridge Univ. Press, 1973.

[9] Ōaku, T., Local solvability of Fuchsian elliptic equations for hyperfunctions, Yokohama Math. J., 36 (1988), 109-114. 
[10] Serra, E., Local solvability for a class of totally characteristic operators, Boll. U.M.I., Ser. VI, Vol. III-C (1984), 131-141.

[11] Tahara, H., Fuchsian type equations and Fuchsian hyperbolic equations, Japan. J. Math. New Ser., 5 (1979), 245-347.

[12] - Singular hyperbolic systems, III. On the Cauchy problem for Fuchsian hyperbolic partial differential equations, J. Fac. Sci. Univ. Tokyo Sect. IA Math., 27 (1980), 465-507.

[13] — Singular hyperbolic systems, IV. Remarks on the Cauchy problem for singular hyperbolic partial differential equations, Japan. J. Math. New Ser., 8 (1982), 297-308.

[14] - On the local solvability of Fuchsian type partial differential equations, Algebraic analysis, Volume II (edited by Kashiwara, M. and Kawai, T.), 837-848. Academic Press, Boston, 1988.

[15] - Solvability in distributions for a class of singular differential operators, I. Proc. Japan Acad., 64 (1988), 219-222.

[16] - Solvability in distributions for a class of singular differential operators, II. Proc. Japan Acad., 64 (1988), 318-321. 